\title{
Ensaio sobre a origem da vantagem competitiva e o desempenho operacional da firma a partir do uso de métricas contábeis
}

\section{Essay on the origin of competitive advantage and performance of the firm from the use of accounting metrics}

Ensayo sobre el origen de la ventaja competitiva y el rendimiento operacional de la firma a partir del uso de métricas contables

\section{Luiz Cláudio Louzada}

Doutor em Administração pelo CEPEAD (UFMG)

Professor-Adjunto do PPGCon (UFES)

Endereço: Rua Diogenes Malacarne, 145, apto. 301

CEP: 29.101-210 - Vila Velha/ES - Brasil

Telefone: (27) 81483939

E-mail: louzadalvi@yahoo.com.br

\section{Márcio Augusto Gonçalves}

Doutor em Administração pela Aston University

Professor-Adjunto do Departamento de Ciências Administrativas (UFMG)

Endereço: Av. Antônio Carlos, 6627 - Prédio FACE - Bloco 01 - $4^{\circ}$ andar, Sala 4098,

Pampulha

CEP: 31.270-901 - Belo Horizonte/MG - Brasil

Telefone: (31) 97220246

E-mail:marciouk@yahoo.com

\section{Bruno Pérez Ferreira}

Doutor em Administração pela Universidade Federal de Minas Gerais (UFMG)

Professor-Adjunto do Departamento de Ciências Administrativas (UFMG)

Endereço: Av. Antônio Carlos, 6627 - Prédio FACE - Bloco 01 - $4^{\circ}$ andar, Sala 4061,

Pampulha

CEP: 31.270-901 - Belo Horizonte/MG - Brasil

Telefone: (31) 34097234

E-mail: brunoperez.bh@gmail.com

Artigo recebido em 29/09/2015. Revisado por pares em 24/062017. Reformulado em 15/10/2017. Recomendado para publicação em 26/08/2017 por Carlos Eduardo Facin Lavarda (Editor-Chefe). Publicado em 31/12/2017. 


\title{
Resumo
}

Este ensaio consiste em uma proposta de investigação da origem da vantagem competitiva da firma a partir do uso de métricas contidas na estrutura das demonstrações contábeis. A origem da vantagem competitiva é segregada em duas dimensões: endógenas, sendo formada por recursos à disposição da firma gerados a partir das escolhas dos gestores; e exógena, configurada por características do setor no qual a firma se insere, como o nível de competição e o dinamismo do setor de atividade. Na origem da vantagem competitiva são variáveis explicativas do desempenho operacional da firma cujas proxies estão contidas na estrutura das demonstrações contábeis. Fundamentos contidos no arcabouço teórico da estratégia e no campo da contabilidade, quando aproximados, sugerem a existência de pesquisas entre campos do conhecimento que permitem investigar o desempenho da firma em seu contexto. Espera-se que a possibilidade de aproximação entre tais campos, (i)contribuir para o aumento da capacidade interpretativa dos indicadores contidos nas demonstrações contábeis quando relativizados ao ambiente em que a firma atua; e, por conseguinte, (ii)contribuir para a utilidade das métricas contábeis como proxies para pesquisas no campo da vantagem competitiva. Assim, este ensaio (iii) sugere as hipóteses e define uma metodologia de pesquisa para investigação da origem da vantagem competitiva com adoção de proxies contidas na estrutura das demonstrações contábeis.

Palavras-chave: Vantagem competitiva; Desempenho operacional da firma; Nível de competição; Grau de dinamismo; Estrutura das demonstrações contábeis

\begin{abstract}
This essay aims to investigate the source of firm competitive advantage from the use of metrics contained on financial statements. In this study, the source of competitive advantage is segregated in two dimensions: endogenous, from the resources available to the company, generated by the managers choices; and exogenous, from characteristics of the industry in which the firm is inserted, such as the competition level and market dynamism. The source of competitive advantage are explained by the firm operating performance variables whose proxies are contained on the financial statements structure. Fundamentals contained on the theoretical framework of strategy and accounting fields, when approximated, suggest the existence of research between such fields of knowledge that allows the investigation of the performance of the firm in its context. We expect this possible approximation of fields of knowledges to contribute to the increase of the interpretative ability of indicators contained in the financial statements when relativized to the environment in which the firm operates; and, therefore, (ii) to contribute to the usefulness of accounting metrics as proxies for research in the field of competitive advantage. Thus, this essay (iii) suggests the hypotheses and defines a research methodology to investigate the source of the competitive advantage with the adoption of proxies contained in the structure of the financial statements.
\end{abstract}

Keywords: Competitive advantage; Operating performance of the firm; Level of competition; Degree of dynamism; Structure of the financial statements

\section{Resumen}

Este ensayo consiste en una propuesta de investigación del origen de la ventaja competitiva de la firma a partir del uso de métricas contenidas en la estructura de las demostraciones contables. El origen de la ventaja competitiva es segregada en dos dimensiones: endógenas, siendo formada por recursos a disposición de la firma generados a partir de las elecciones de los gestores; y exógena, configurada por características del sector en el cual la firma se inserta, como el nivel de competición y el dinamismo del sector de actividad. El origen de la ventaja competitiva son variables explicativas del desempeño operacional de la firma cuyas proxies están contenidas en la estructura de las demostraciones contables. Fundamentos contenidos en el marco teórico de la estrategia y en el campo de la contabilidad, cuando aproximados, sugieren la existencia de investigaciones entre campos del conocimiento que permiten investigar el desempeño de la firma en su contexto. Se espera que la posibilidad de aproximación entre tales campos, (i) contribuya al aumento de la capacidad interpretativa de los indicadores contenidos en las demostraciones contables cuando estén relativizados al ambiente en que la firma actúa; $y$, por consiguiente, (ii) contribuir a la utilidad de las métricas contables como proxies para investigaciones en el campo de la ventaja competitiva. Así, este ensayo (iii) sugiere las hipótesis y define una metodología de investigación para el estudio del origen de la ventaja competitiva con la adopción de proxies contenidas en la estructura de las demostraciones contables.

Palabras clave: Ventaja competitiva; Rendimiento operacional de la firma; Nivel de competición; Grado de dinamismo; Estructura de las demostraciones contables 


\section{Introdução}

Estudos empíricos baseiam-se na observação de variáveis do desempenho para medir a competitividade das firmas sob o argumento de que a vantagem competitiva é um construto precedente lógico do desempenho superior (POWELL, 2001; WIGGINS e RUEFLI, 2002; VASCONCELOS e BRITO, 2004). Essas pesquisas fundamentam-se no discurso determinístico da organização industrial e da visão baseada em recursos. De acordo com o modelo de organização industrial, o setor industrial em que a firma escolhe atuar tem uma forte influência sobre o desempenho (PORTER, 1979, 1983; SCHERER e ROSS, 1990; BANKER e outros, 2014). A teoria da visão baseada em recursos pressupõe que cada empresa é um conjunto de recursos e capacidades (WERNERFELT, 1984; BARNEY, 1991, 2001; ALLEN e HELMS, 2006). Em comum, tais abordagens fornecem a base para a geração da vantagem competitiva da firma sugerindo, em suas particularidades, que a origem da vantagem competitiva é endógena e/ou exógena à firma, e abordam a existência de uma relação causal entre a vantagem competitiva e o desempenho da firma.

Questões relacionadas ao estudo do desempenho fazem parte dos campos de estudo da estratégia e da contabilidade, que se diferenciam no foco do interesse e na unidade de análise. Enquanto a estratégia volta-se para a análise das escolhas, das decisões e dos recursos inerentes ao ambiente da firma (RUMELT, SCHENDEL, TEECE, 1991), a análise das demonstrações contábeis fornece métricas financeiras, operacionais e econômicas que, combinadas, têm como proposição o estudo do desempenho da firma sob uma perspectiva ex-post-facto, permitindo a sustentação ou o reposicionamento das escolhas e das decisões dos gestores no uso de recursos (PALEPU e HEALY, 2008; OHLSON, 2014).

A aproximação entre tais campos do conhecimento poderia contribuir para a identificação de indicadores contábeis como proxies da vantagem competitiva para investigar o desempenho da firma, enfatizando-se que a análise das informações contábeis, combinada com o contexto no qual a firma se insere, permite ao analista entendimentos mais relevantes do desempenho da firma.

Portanto, ao considerar que a origem da vantagem competitiva, numa dimensão endógena, pode ser configurada como recursos à disposição da firma e evidenciada por métricas contidas na estrutura das demonstrações contábeis a partir de indicadores patrimoniais, financeiros, operacionais, de estrutura e origem de financiamento (como proxies da vantagem competitiva, numa dimensão de recursos); e que de forma complementar a vantagem competitiva também pode ser originada numa dimensão exógena, a partir de características ambientais como níveis de competição e previsibilidade do setor nas quais a firma se insere, possibilitando ser relacionado ao desempenho da firma, procurar-se-á, no transcorrer deste ensaio, sustentar uma relação teórica para se investigar a seguinte questão $\left(Q_{1}\right)$ : Como estabelecer relação teórica entre a origem da vantagem competitiva e o desempenho operacional da firma e o uso de métricas contidas na estrutura das demonstrações contábeis como proxies para investigação origem da vantagem competitiva?

O objetivo deste ensaio consiste em buscar sustentações teóricas para relacionar as dimensões da vantagem competitiva e o desempenho da firma a partir de proxies contidas nos demonstrativos contábeis. Os objetivos específicos para o desenvolvimento deste trabalho, $a$ priori, são os seguintes: $\boldsymbol{O}_{1}$ : verificar se a origem da vantagem competitiva pode ser relacionadas a fatores endógenos ou exógenos à firma utilizando métricas contidas na estrutura das demonstrações contábeis; $\boldsymbol{O}_{2}$ : desenvolver e sustentar hipóteses de pesquisas com base na relação dos fundamentos da vantagem competitiva e das métricas contidas na estrutura das 
demonstrações contábeis; $\boldsymbol{O}_{3}$ : propor uma metodologia de teste para investigação da origem da vantagem competitiva a partir da adoção de proxies contábeis.

Espera-se encontrar uma aproximação entre a vantagem competitiva e as métricas contidas nas demonstrações contábeis contribuindo para a avaliação do desempenho da firma numa dimensão de coexistência entre a firma e o seu ambiente, potencializando, dessa forma, a evidenciação das informações contidas nas demonstrações contábeis e oferecendo métricas para monitorar o desempenho da firma numa dimensão ex-post-fato. Especificamente, identificar (i) a utilidade das demonstrações contábeis no contexto da tomada de decisões; (ii) contribuir para o desenvolvimento e para a definição de proxies e de métricas contábeis para monitoramento e estudos da estratégia e da vantagem competitiva, além de (iii) buscar uma relação (aproximação) entre esses campos do conhecimento.

\section{Referencial Teórico}

\subsection{A Vantagem Competitiva}

A vantagem competitiva é tema central do campo de estratégia e uma das hipóteses mais utilizadas para explicar a heterogeneidade do desempenho entre as firmas (SOUTH, 1981). O estudo da relação causal entre a origem da vantagem competitiva e o desempenho da firma pode ser originado não só dos recursos específicos da firma, mas também da posição que ela ocupa no mercado (CAVES, 1984). As principais correntes teóricas em estratégia buscam explicar a competitividade da firma, (i) pela escolha e pelo posicionamento em um dado setor industrial (PORTER, 1983), (ii) pelo desenvolvimento e pela apropriação de recursos (BARNEY, 1991) ou ainda (iii) pelo desenvolvimento de rotinas e pela sua capacidade dinâmica (TEECE; PISANO; SHUEN, 1997; BRITO; 2005).

Em comum, essas teorias argumentam a existência de uma vantagem competitiva como a principal explicação para a heterogeneidade do desempenho. A estrutura do setor é abordada como um determinante exógeno, enquanto os recursos da firma, combinados com sua capacidade dinâmica, são considerados determinantes endógenos à firma para a geração da vantagem competitiva.

\subsubsection{A Vantagem Competitiva e a Teoria do Posicionamento}

Um dos modelos conceituais mais difundidos para a análise da vantagem competitiva é o modelo Estrutura-Conduta-Desempenho, da nova organização industrial (SCHERER e ROSS, 1990). A essência do paradigma da Organização Industrial consiste em relacionar o desempenho da firma com variáveis dependentes das características do ambiente ao qual ela está exposta (BAIN, 1964; PORTER, 1983), conforme ilustrado na Figura 1.

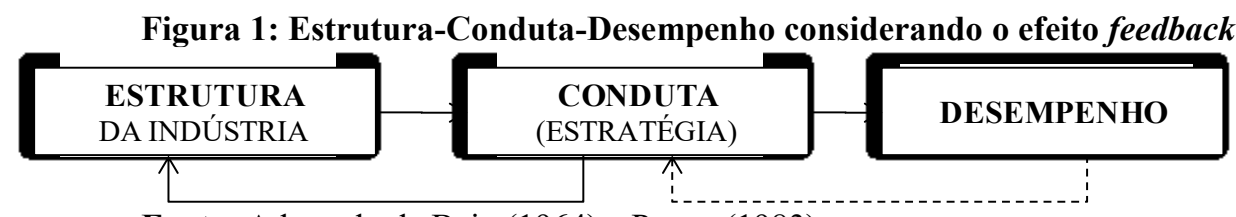

Fonte: Adaptado de Bain (1964) e Porter (1983)

O modelo Estrutura-Conduta-Desempenho supõe que o desempenho econômico das firmas é o resultado direto de seu comportamento concorrencial, em termos de fixação de preços e de custos, e esse comportamento depende da estrutura da indústria na qual as firmas estão inseridas (VASCONCELOS e CYRINO, 2000). Scherer e Ross (1990) comentam que: 
- O desempenho é consequência das condutas ou dos comportamentos dos vendedores e dos compradores, das práticas e das políticas de determinação de preços, da cooperação tácita entre firmas, da linha de produtos e estratégias de divulgação, dos gastos com pesquisa e desenvolvimento, dos investimentos em técnicas de produção, das táticas legais e assim por diante;

- A conduta, ou o comportamento, depende da estrutura predominante do mercado, caracterizada pelo número e pelo tamanho dos ofertantes e dos demandantes (concentração), pela presença ou ausência de barreiras de entrada de novas firmas, pelas formas das curvas de custo, pelo grau de integração vertical das firmas, entre outros fatores; $\mathrm{e}$

- A estrutura de mercado é influenciada por características básicas que atuam tanto pelo lado da oferta quanto pelo da demanda. Pela oferta há, por exemplo, localização da matéria-prima, grau de tecnologia, durabilidade do produto, relação valor/peso e atitudes nos negócios.

O posicionamento da firma consiste em um determinante de seu desempenho. O posicionamento adequado é capaz de gerar retornos para a firma acima da média da indústria, mesmo que a estrutura industrial seja desfavorável e o desempenho médio da indústria seja, portanto, modesto (BAIN, 1964; PORTER, 1979).

\subsubsection{A Vantagem Competitiva e a Teoria de Recursos}

Foss (1997) afirma que a competitividade de uma organização se fundamenta essencialmente em sua capacidade de selecionar e de combinar recursos excludentes e complementares. A teoria dos recursos está fundamentada em duas generalizações empíricas e em dois postulados, sendo: (i) as generalizações empíricas: existem diferenças sistemáticas entre as firmas, no tocante à forma com que elas controlam os recursos necessários para a implementação de suas estratégias, e tais diferenças são relativamente estáveis; (ii) e os postulados: as diferenças nas dotações de recursos causam diferenças de desempenho, e as firmas têm por prática constante maximizar o desempenho econômico.

Essa percepção provoca uma alteração relevante da visão sobre a natureza da concorrência. Em vez de ser uma concorrência entre produtos ou serviços, passa a ser uma concorrência entre recursos e competências (HAMEL, 1994). Portanto, se a vantagem competitiva pressupõe que os recursos das firmas são heterogêneos, é esperado que os seus diferentes recursos causem diferenças em seu desempenho operacional (COMBS, CROOK, SHOOK, 2005).

\section{CONTABILIDADE E VANTAGEM COMPETITIVA}

Assumindo-se que as informações econômicas e financeiras são as principais referências para a tomada de decisões no contexto empresarial e, considerando-se as demonstrações contábeis uma de suas principais fontes (PALEPU; HEALY, 2008), torna-se relevante o estudo da relação entre vantagem competitiva e desempenho operacional da firma com base no uso de métricas contidas na estrutura das demonstrações contábeis.

Com esse propósito, na Figura 2 busca-se ilustrar a relação entre esses campos do conhecimento. Sustenta-se, no argumento, que se houver proximidade e consistência teórica nessa relação, espera-se que os construtos teóricos da estratégia, como o modelo Estrutura- 
Conduta-Desempenho e os conceitos de vantagem competitiva e os fundamentos que formam as métricas contidas nas demonstrações contábeis, também possam ser relacionados.

Figura 2: Relação teórica entre os campos do conhecimento

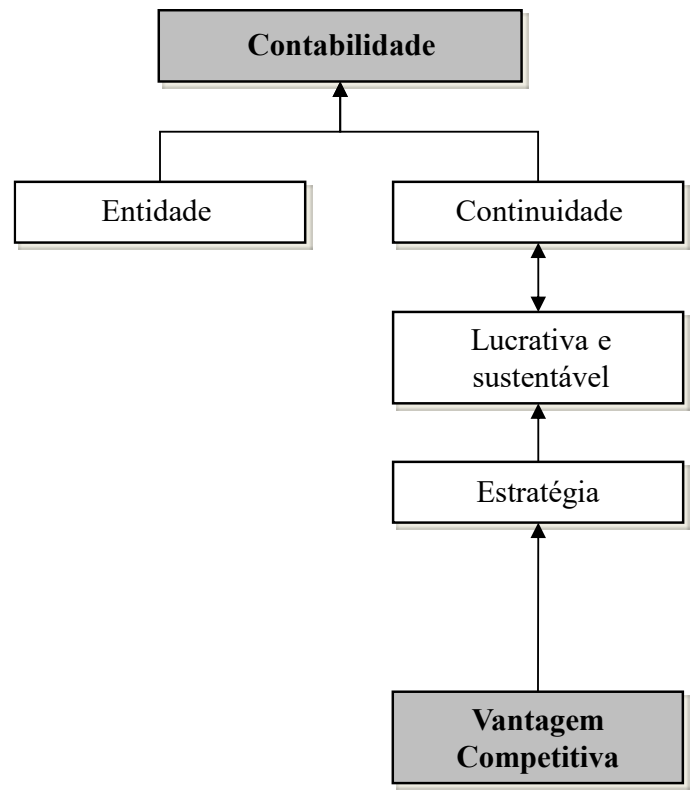

Postulado que se refere ao ambiente no qual as entidades atuam e às formas com que operam neste mercado (HENDRICKSEN, 1999).

As empresas por meio da estratégia competitiva, buscam definir e estabelecer uma abordagem para a competição em sua indústria que seja, ao mesmo tempo, lucrativa e sustentável (PORTER, 1986).

A vantagem competitiva relaciona-se com a capacidade de criação de valor acima da média de seus concorrentes que é inferida a partir da análise do desempenho relativo de uma unidade empresarial em relação ao mercado onde esta se insere (BARNEY, 2003).

Fonte: Louzada (2004) com adaptações para a vantagem competitiva.

Se os postulados ${ }^{i}$ contábeis são definidos como suposições derivadas do ambiente econômico (HENDRIKSEN, 1999), que sustentam o arcabouço teórico da Contabilidade influenciando a forma de mensuração, a configuração e as informações contidas em seus relatórios, então, as demonstrações contábeis deveriam ser capazes de refletir os aspectos da estratégia da firma, visto que tanto as teorias de estratégia quanto as teorias contidas no arcabouço da contabilidade evidenciam, em suas formas particulares, que a continuidade da firma pressupõe lucratividade e sustentabilidade ao longo do tempo. É neste ponto que os postulados contábeis parecem guardar um alinhamento (aproximação) com as premissas do arcabouço conceitual da estratégia e da vantagem competitiva, no contexto da estrutura e das características de mercado.

Neste ponto, cabe estabelecer uma razoável relação entre as demonstrações contábeis e a estratégia. A Contabilidade é uma linguagem que mensura as variações patrimoniais da firma, procurando evidenciar o desempenho em um determinado intervalo de tempo, configurando-se em um instrumento para o estudo da eficiência na alocação de recursos. Daí a sua importância numa economia de mercado. Na ausência de informação apropriada, o risco aumenta e, devido ao custo de capital, haverá um reflexo nos preços (LIMEIRA e outros, 2003). Portanto, a utilidade da informação contábili ${ }^{\mathrm{ii}}$ está vinculada ao suporte para a efetivação de contratos e a comprovação das relações estabelecidas, e é fundamental o controle das atividades econômicas na avaliação do desempenho da firma.

$\mathrm{O}$ arcabouço teórico da contabilidade fundamenta-se em seus postulados para alcançar seus objetivos de tornar as atividades das firmas compreensíveis, e atender a seu propósito de tornar possível para seus usuários alcançar um julgamento calculado do sucesso da firma na 
execução de suas atividades (CHAMBERS, 1966). O conteúdo da informação contábil serve a duas funções: (i) facilitar decisão e (ii) controlar comportamentos. O processo de geração e de divulgação das demonstrações contábeis está apoiado na utilidade da informação (ZIMMERMAN, 1997).

Assim, relacionar métricas contidas nas demonstrações contábeis a serem adotadas como proxies para pesquisas da vantagem competitiva, poderia contribuir, sobremaneira, numa tentativa de aproximação e para o desenvolvimento de novas pesquisas entre a vantagem competitiva e a utilização de métricas das demonstrações contábeis de forma a se investigar o desempenho da firma.

\section{Desenvolvimento de Hipóteses de Pesquisa}

Os recursos da firma são evidenciados nos balanços e dispostos, segundo a lógica do modelo contábil, como ativos financeiros, operacionais e de estrutura (dimensão endógena), que se configuram a partir das escolhas dos gestores no contexto da firma. As características ambientais, como o nível de concorrência e o grau de dinamismo do ambiente (dimensão exógena), também interferem no desempenho da firma. Os reflexos das variáveis endógenas e exógenas interferem na formação e na diferença entre preço e custo (apropriação de valor), evidenciada no desempenho operacional da firma. Tal efeito sustenta o desenvolvimento das seguintes hipóteses de pesquisa e suas interações, conforme Figura3.

Figura 3: Relacionando fundamentos da vantagem competitiva a de métricas contábeis.

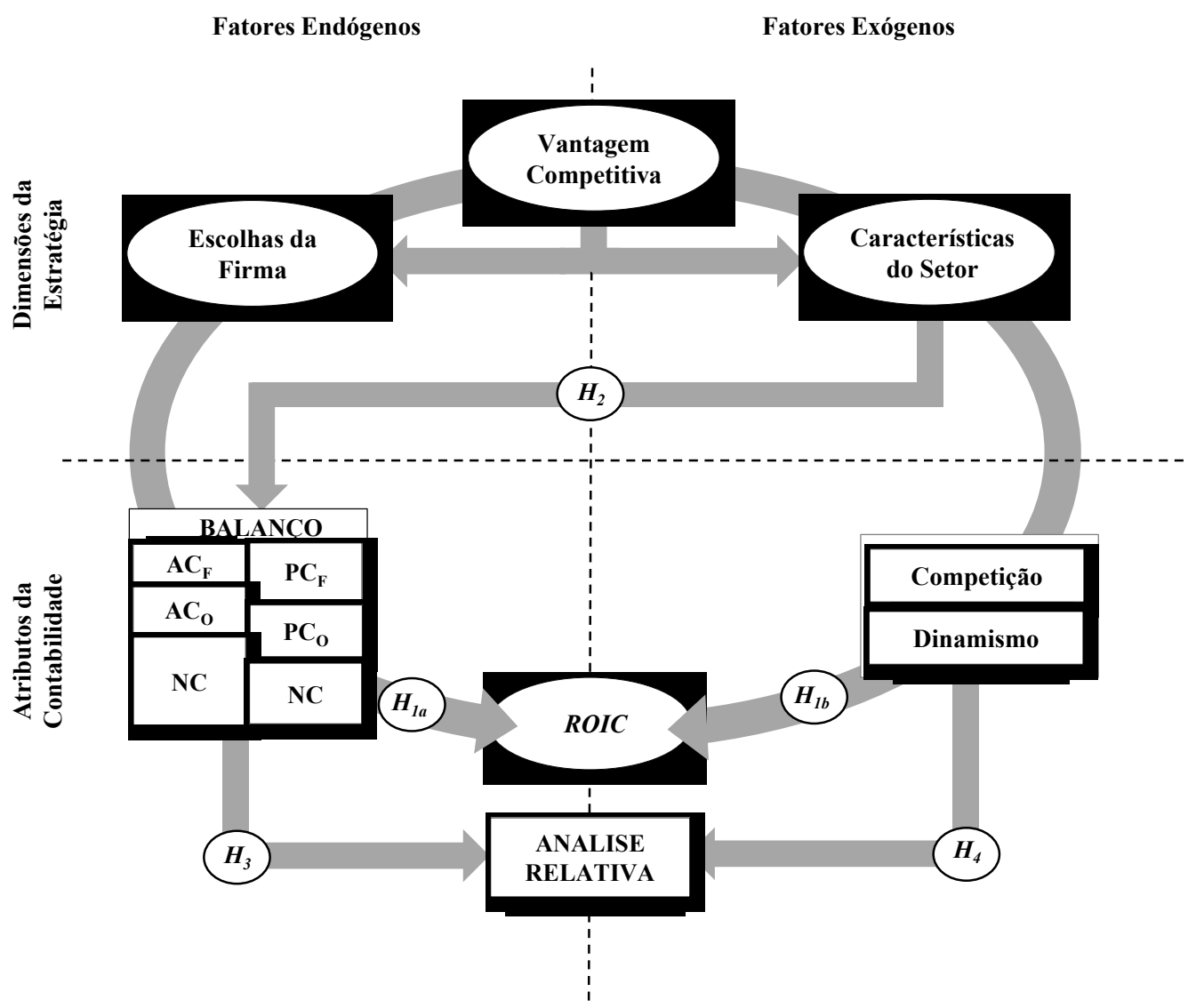

Fonte: $\mathrm{O}$ autor, fundamentado nas revisões dos itens 2 e 3 deste ensaio. 
Se a vantagem competitiva pressupõe que os recursos das firmas são heterogêneos, é esperado que, devido a essa heterogeneidade de recursos, as firmas apresentem diferenças de desempenho econômico entre si (HAMEL, 1994; BANKER e outros, 2011). E, em condições de interação entre origens da vantagem competitiva, características da estrutura patrimonial da firma (endógenas) e características do setor em que a firma se insere (exógenas), definem-se as seguintes hipóteses conjuntas relativas à origem da vantagem competitiva $\boldsymbol{H}_{1 \boldsymbol{a}}$ e $\boldsymbol{H}_{\boldsymbol{l}}$ :

Assim, $\mathrm{H}_{1 a}$ : As escolhas feitas pelos gestores são captadas pelo modelo da contabilidade na estrutura patrimonial na forma de ativos financeiros, operacionais, de estrutura e de fonte de captação de recurso. Portanto, podem ser adotadas como variáveis endógenas da firma, podendo assim, explicar o desempenho operacional da firma.

Por outro lado, e de forma complementar,

$H_{1 b}:$ As características do ambiente no qual a firma está inserida, como níveis diferenciados de concorrência e graus diferentes de dinamismo do ambiente, podem ser adotadas como variáveis exógenas para explicar o desempenho operacional da firma.

Por conseguinte, considerando-se que os fatores exógenos podem influenciar as variáveis endógenas da firma, configura-se a seguinte hipótese de interação entre as variáveis:

Assim, $\mathrm{H}_{2}:$ As variáveis exógenas interferem na relação entre fontes endógenas de vantagem competitiva $e$ desempenho operacional da firma, ou seja, as características ambientais afetam a relação causal entre a estrutura patrimonial $e$ o desempenho operacional da firma evidenciado na estrutura das demonstrações contábeis.

Dada a existência de relação teórica entre as relações contidas nas hipóteses acima, e estabelecendo-se uma análise relativa das informações contidas nas demonstrações contábeis da firma com seu setor, formulam-se as demais hipóteses a seguir.

Vantagens competitivas nada mais são do que assimetrias em relação aos concorrentes. A intensidade das forças de mercado é função da estrutura da indústria ou das características econômicas e técnicas (PORTER, 1983; FARINA, 1999; COFF, 2010).

Assim, $H_{3}:$ A comparação entre estrutura patrimonial da firma (financeira, operacional, de estrutura e fontes de recursos) e aquela do setor em que a empresa se insere em relação com o desempenho operacional da firma.

O ambiente no qual a organização está inserida pode afetar a formulação e a implantação da estratégia. Organizações inseridas em ambientes dinâmicos e competitivos sofrem maior pressão para as revisões de estratégias (ANDREWS, 1996). Nessa lógica, a incerteza é a variável mais comumente citada para caracterizar o ambiente externo de uma organização.

Ademais, a análise do desempenho pode demonstrar os direcionamentos e as tendências gerenciais, derivados da capacidade de o gestor fazer escolhas sobre processos conflitantes e sobre incertezas no ambiente, e a análise da investigação dos resultados deve compreender a influência das decisões estratégicas evidenciadas no desempenho da gestão da firma (RUMELT, SCHENDEL, TEECE, 1991), provocados por características do setor em que a firma se insere.

Assim, $H_{4}:$ A relação entre o desempenho operacional e a estrutura patrimonial da firma e do setor em que ela atua é moderada por diferentes níveis de competição e grau de dinamismo do setor.

Essa convergência de fatores endógenos e exógenos para a explicação/sustentação da origem do conceito da vantagem competitiva sugere que a investigação possa gerar espaços a fim de as disciplinas do campo da análise das demonstrações contábeis e da vantagem 
competitiva poderem se enriquecer mutuamente, o que aumenta a relevância da investigação e do teste das hipóteses definidas.

\section{Metodologia}

\subsection{Variáveis de Pesquisa no Modelo de Regressão}

A estrutura básica do modelo de regressão para dados em painel a ser adotada em uma pesquisa, conforme sugerido, seria:

$$
\begin{aligned}
& \boldsymbol{R O I C}_{i t}=\beta_{0}+\beta_{1} \boldsymbol{A C F}_{i t}+\beta_{2} \boldsymbol{P C F _ { i t }}+\beta_{3} \boldsymbol{A C O}_{i t}+\beta_{4} \mathbf{P C O}_{i t}+\beta_{5} \boldsymbol{A N} \boldsymbol{C}_{i t}+\beta_{6} \boldsymbol{P N C}_{i t} \\
& +\beta_{7} \boldsymbol{A L} \boldsymbol{A}_{i t}+\beta_{8} \boldsymbol{H} \boldsymbol{H} \boldsymbol{I}_{i t}+\beta_{9} \boldsymbol{D I} \boldsymbol{N}_{i t}+\beta_{10} \boldsymbol{H H I} * \boldsymbol{D I I} \boldsymbol{N}_{i t}+\alpha_{i}+u_{i t}
\end{aligned}
$$

em que $i=1, \ldots, 679$ firmas brasileiras; $t=1, \ldots, 71$ trimestres, referentes ao período de março de 1999 a setembro de 2013; ROIC: retorno operacional da firma $i$, no trimestre $t ; \beta_{0}$ : é o intercepto do modelo; $\beta$ : representa os coeficientes angulares estimados para cada variável independente; $\alpha_{i}+u_{i t}$ : são os erros do modelo, $\alpha_{i}$ indica o efeito individual específico não observável e $u_{i t}$ varia com as unidades e com tempo, é o de erro "usual" da regressão. As variáveis do modelo são apresentadas no quadro 1.

\begin{tabular}{|c|c|c|c|}
\hline & Tipo & Variáveis & Fórmula \\
\hline \multirow{8}{*}{ 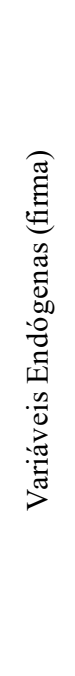 } & Des empenho & Retorno sobre o capital investido (ROIC) & $R O I C=\frac{N O P A T}{\text { Investimentos }}$ \\
\hline & \multirow{2}{*}{ Financeiro } & Ativo circulante financeiro (ACF) & $A C F=\frac{\text { Ativo Circulante Financeiro }}{\text { Total do Ativo }}$ \\
\hline & & Passivo circulante financeiro (PCF) & $P C F=\frac{\text { Passivo Circulante Financeiro }}{\text { Passivo }+P L}$ \\
\hline & \multirow{2}{*}{ Operacional } & Ativo circulante operacional (ACO) & $A C O=\frac{\text { Ativo Circulante Operacional }}{\text { Total do Ativo }}$ \\
\hline & & Passivo circulante operacional (PCO) & $P C O=\frac{\text { Passivo Circulante Operacional }}{\text { Passivo }+P L}$ \\
\hline & \multirow{2}{*}{ Permanente } & Ativo não circulante (ANC) & $A N C=\frac{\text { Ativo não circulante }}{\text { Total do Ativo }}$ \\
\hline & & Passivo não circulante (PNC) & $P N C=\frac{\text { Passivo não circulante }}{\text { Passivo }+P L}$ \\
\hline & $\begin{array}{l}\text { Origem } \\
\text { dos recursos }\end{array}$ & Alavancagem $(\mathbf{A L A})$ & $A L A=\frac{\text { Capital de Terceiros }}{\text { Passivo }+P L}$ \\
\hline \multirow{2}{*}{ 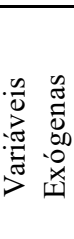 } & $\begin{array}{l}\text { Concentração } \\
\text { (competição) }\end{array}$ & Herfindahl $(\boldsymbol{H} \boldsymbol{H I})$ & $H H I=\sum_{i=1}^{n} \beta_{i}^{2} \quad \beta_{i}=\frac{Q_{i}}{\sum_{i=1}^{M} Q_{i}}$ \\
\hline & $\begin{array}{l}\text { Imprevisibilidade } \\
\text { e incerteza }\end{array}$ & Dinamismo $(\boldsymbol{D I N})$ & $Y_{t}=\beta_{0}+\beta_{1} t+u_{t}$ \\
\hline
\end{tabular}

Quadro 1: Variáveis endógenas e exógenas definidas a partir de proxies contábeis

Fonte: O autor

\subsection{Seleção da Amostra e Tratamento dos Dados}

A amostra poderia ser extraída do banco de dados da Economática ${ }^{\circledR}$, Comdinheiro ${ }^{\circledR}$ ou Thompson ${ }^{\circledR}$ empresas especializadas em informações para o mercado de capitais. Os dados referem-se às empresas que têm (ou tiveram) suas demonstrações contábeis publicadas no período determinado, em períodos anuais, considerando a maior disponibilidade dos dados. 
Sendo selecionadas todas as empresas listadas na Bovespa de todos os setores (exceto bancos e fundos e outros que foram que seriam excluídas da amostra, dada a diferença na estrutura de formação das demonstrações em relação aos outros setores de atividade) e, em seguida, essas empresas devem ser agrupadas de acordo com a classificação da North American Industry Classification System (NAIC), sendo excluídas das amostras aquelas que não apresentavam os seguintes dados a cada trimestre observado: total do ativo, ativo circulante financeiro, ativo circulante operacional, ativo não circulante, total do passivo mais patrimônio líquido, passivo circulante financeiro, passivo circulante operacional, passivo não circulante, patrimônio líquido, receita bruta de vendas e ROIC.

\subsection{Modelo Econométrico}

Para a especificação do modelo de dados em painel a ser utilizado, foi estabelecido o seguinte passo a passo, conforme Figura 4.

Figura 4: Esquema para aplicação dos testes e para a estimação do modelo

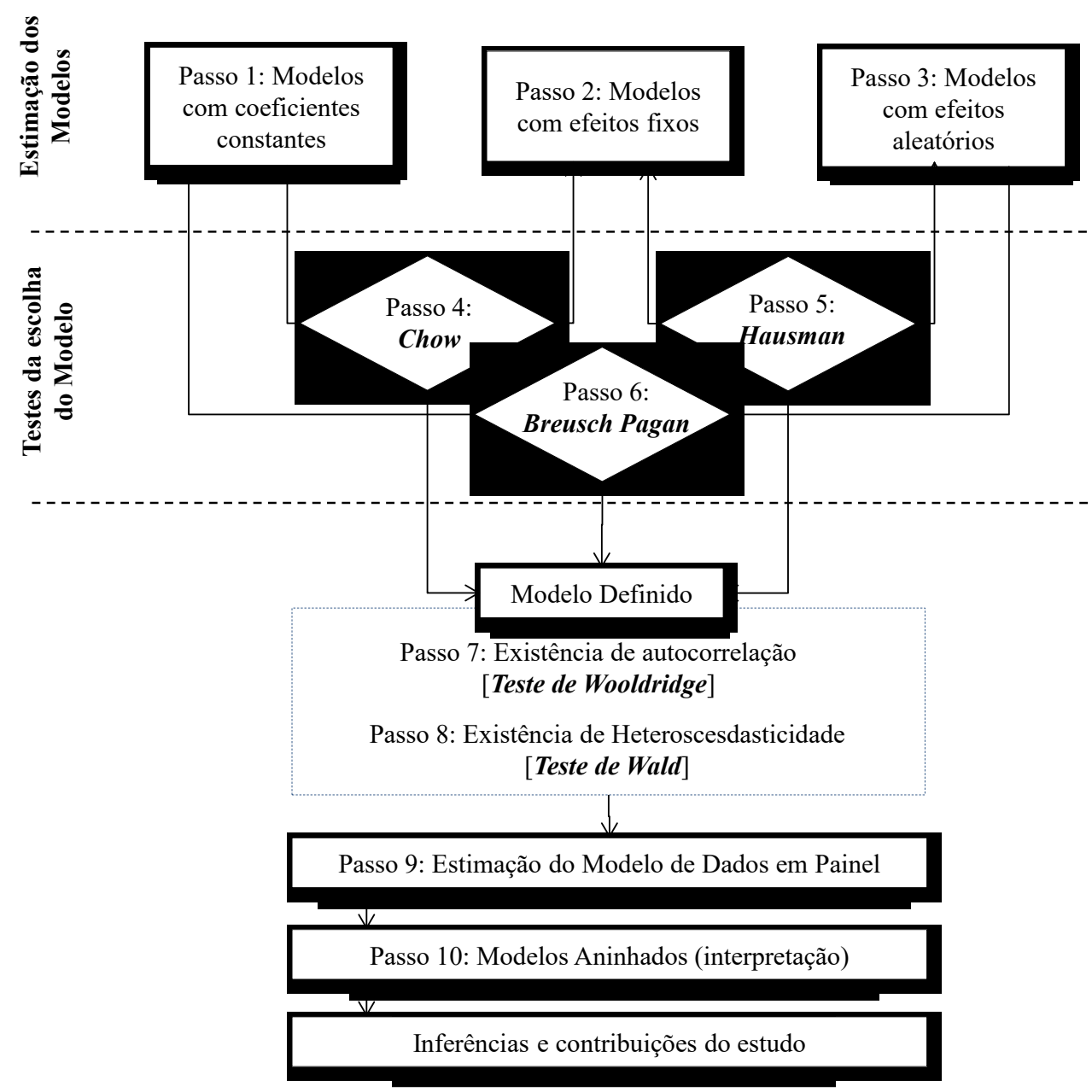

\subsection{Discussões e Análise dos Resultados}

Os modelos aninhados são adequados para explicar a variação de fenômenos decorrentes da combinação de fatores, nesse caso, o estudo de fatores endógenos e exógenos à 
firma que se relacionam com o desempenho operacional a partir de proxies das demonstrações contábeis de empresas que operam no mercado brasileiro. O modelo definido no item 5.1 será analisado simultaneamente, com o objetivo de verificar as hipóteses definidas para esta pesquisa.

Comparações entre os modelos restritos e irrestritos poderão ser adequados para capturar o efeito marginal da presença, ou não, das variáveis nos modelos. As variáveis também poderiam ser reagrupadas em variáveis endógenas e exógenas a firma para capturar o efeito causal que a firma e a indústria causam no desempenho operacional da firma.

Por fim, efeitos moderadores ou mediadores poderiam integrar o modelo menos restrito a partir de interações entre as variáveis explicativas, com o propósito de identificar o efeito moderador e/ou mediador que as variáveis exógenas podem causar na relação entre o grupo de variáveis endógenas e o desempenho operacional da firma.

\section{Considerações Finais}

Drnevich, Madsen e Newbert (2010) destacam que o tema sobre pesquisas recentemente retomado no painel sobre vantagem competitiva em 2010 na Academy of Management resultou em "várias questões importantes para estudo da vantagem competitiva as quais permanecem sem resposta", tais como: Como podemos efetivamente retratar, modelar e medi-la? Como as dinâmicas organizacional, competitiva e ambiental a afetam? Como ela se desenvolve? Conforme Brito e Brito (2012). Este ensaio configura-se em uma possibilidade de respostas das questões acima com a adoção de métricas contidas nas demonstrações contábeis.

Embora os principais fundamentos adotados neste ensaio não sejam novos, sua originalidade está na forma como sugere relacionar campos teóricos distintos, propondo a utilização de variáveis patrimoniais como proxies da vantagem competitiva, e estabelecer relação com a variável desempenho operacional da firma, utilizando métricas contidas na estrutura das demonstrações contábeis.

Espera-se que essa pesquisa contribua para aumentar o conhecimento sobre a vantagem competitiva e sua importância dos estudos da decomposição e origem do desempenho operacional da firma a partir de proxies contida na estrutura das demonstrações contábeis. Viabilizando assim, investigar/identificar fatores dentro de cada indústria e como se relacionam, e o efeito sobre o desempenho da firma, gerando uma compreensão da explicação da variância do desempenho da firma e o efeito provocado pelo setor. Do ponto de vista prático, a identificação de fatores que mais contribuem para o desempenho da firma possibilita aos gestores focarem sua atenção em fatores com maior relevância no desempenho.

\section{Referências}

ALLEN, R. S.; HELMS, M. M. Linking strategic practices and organizational performance to Porter's generic strategies. Business Process Management Journal. v. 12, n. 4, p. 433-454, 2006 (DOI 10.1108/14637150610678069)

ANDREWS Kenneth R. The concept of corporate strategy of strategic management. In: MCKIERNAM, Peter. Historical evolution V.I. Bookfeld, Dartmouth Publishing Company, p. 15-44, 1996.

BAIN. J. S. The Impact on Industrial Organization. The American Economic Review, v.54, no 3, p. 28-32, May, 1964. 
BANKER, R. D.; MASHRUWALA, R.; TRIPATHY, A. Does a differentiation strategy lead to more sustainable financial performance than a cost leadership strategy? Management Decision, v. 52, p. 872-896, 2014. (DOI: 10.1108/MD-05-2013-0282)

BANKER, R. D.; HU, N.; PAVLOU, A. P.; LUFTMAN, J. CIO Reporting structure, strategic positioning, and firm performance. MIS Quartely research article. v. 35. n. 2, p. 487-504, jun 2011.

BARNEY J. Resource-based theories of competitive advantage: a ten-year retrospective on the resource based view. Strategic Management Journal n² 27, p. 643-650, 2001. (DOI: $10.1177 / 014920630102700602)$

BARNEY, J. B. Firm resources and sustained competitive advantage. Journal of Management, v.17, nº 1, p. 99-120, 1991. (DOI: 10.1177/014920639101700108)

BRITO, L. A.L. Os componentes da variância da taxa de crescimento da firma. 2005. 238 f. Tese (Doutorado em Administração de Empresas) - Escola de Administração de Empresas de São Paulo, Fundação Getúlio Vargas, São Paulo, 2005.

BRITO, Renata Peregrino de; BRITO, Luiz Artur Ledur. Vantagem competitiva e sua relação com o desempenho: uma abordagem baseada em valor. Revista de Administração Contemporânea, v. 16, n. 3, p. 360-380, mai./jun. 2012.

BYRNS, R.; STONE JUNIOR,G. W. Microeconomia. São Paulo: Makron Books, 1996. CAVES, R. E. Economic analysis and the quest for competitive advantage. American Economic Review, v.74, n² 2, p. 127, 1984.

CHAMBERS, Raymond J. Accounting, evaluation and economic behavior. Texas: Scholars Book, 1966.

COFF, R. W. The coevolution of rent appropriation and capability development. Strategic Management Journal, v.31, no 7, p. 711-733, 2010. (DOI: 10.1002/smj.844)

COMBS, J. G.; CROOK, T. R.; SHOOK, C. L. The dimension of organizational performance and its implications for strategic management research. In: D. J. KETCHEN e D. D. BERGH (Orgs.), Research Methodology in Strategy and Management, San Diego, Elsevier, p.259286, 2005. (DOI: 10.1016/S1479-8387(05)02011-4)

FARINA, E. M. M. Q.; AZEVEDO, P. F. de; SAES, M. S. M. Competitividade: mercado, estado e organizações. Editora Singular, São Paulo, 1997.

FOSS, N. J. Research in strategy, economics and Michael Porter. Journal of Management Studies, v.33, no 1, p. 1-24, 1996. (DOI: 10.1111/j.1467-6486.1996.tb00796.x)

FOSS, N. J. Resources, firms and strategies: a reader in the resource-based perspective. New York: Oxford University Press, p. 3-18, 1997.

HAMEL, G. The concept of core competence. Competence-based competition. Chichester: John Willey \& Sons, 1994. 
HENDRIKSEN, Eldon S.; VAN BREDA, Michael F. Teoria da contabilidade. 5. ed. São Paulo: Atlas, 1999.

LOUZADA, Luiz Cláudio. Relação entre barreiras de entrada e o retorno empresarial no mercado brasileiro a partir de dados das demonstrações contábeis. 2004. $142 \mathrm{f}$.

Dissertação (Mestrado em Ciências Contábeis) - Programa de Pós-Graduação em Ciências Contábeis, Fundação Instituto Capixaba de Pesquisas em Contabilidade, Economia e Finanças (FUCAPE), Vitória, 2004.

OHLSON, J. A. An overview. China Journal of Accounting Research. v. 7, p. 65-80, 2014. (DOI.10.1016/j.cjar.2014.03.003)

PALEPU, K. G.; HEALY P. M. Business Analysis \& Valuation: Using Financial Statements, $4^{\circ}$ ed. Thomson South-Western, 2008.

PHILIPS, Edward G. The accretion concept of income. The Accounting Review.v.38, n. 1, p. 14-25, jan. 1963.

PORTER, Michael E. Industrial organization and the evolution of concepts for strategic planning: the new learning. Managerial and Decision Economics, v.4, n 3, p. 172-180, set. 1983. (DOI: $10.1002 / \mathrm{mde} .4090040307$ )

PORTER, Michael E. The structure within industries and companies' performance. The Review of Economics and Statistics, v.61, n. 2, p. 214-227, may. 1979. (DOI: $10.2307 / 1924589)$

POWELL, T. C. Competitive advantage: logical and philosophical considerations. Strategic Management Journal, v.22, n. 9, p. 875-888, 2001. (DOI: 10.1002/smj.173)

RUMELT, R. P.; SCHENDEL, D. E.; TEECE, D. J. Strategic management and economics. Strategic Management Journal, v.12, special issue, p. 5-29, 1991. (DOI: 10.1002/smj.4250121003)

SCHERER, F. M; ROSS, D. Industrial market structure and economic performance. EUA: Houghton Mifflin Company, 1990.

SOUTH, S. E. Competitive advantage: the cornerstone of strategic thinking. The Journal of Business Strategy, v.1, no 4, p.15, 1981. (DOI:10.1108/eb038908)

TEECE, D. J.; PISANO, G. P.; SHUEN, A. Dynamic capabilities and strategic management. Strategic Management Journal, v.18, n. 7, p. 509-533, 1997.(DOI: 10.1002/(SICI)10970266(199708))

VASCONCELOS, F. C.; BRITO, L. A. L. Vantagem competitiva: o construto e a métrica. Revista de Administração de Empresas, v.44, n. 2, p. 70-82, 2004. 
VASCONCELOS, Flávio C.; CYRINO, Álvaro B. Vantagem competitiva: os modelos teóricos atuais e a convergência entre estratégia e teoria organizacional. Revista de Administração de Empresas, v.40, n. 4, p. 20-37, out./dez. 2000. (DOI.org/10.1590/S0034)

WERNERFELT, B. A resource-based view of the firm. Strategic Management Journal, ${ }^{\circ}$ 5, p.171-180, 1984. (DOI: 10.1002/smj.4250050207)

WIGGINS, R. R.; RUEFLI, T. W. Sustained competitive advantage: temporal dynamics and the incidence and persistence of superior economic performance. Organization Science, v.13, no 1, p. 81-105, 2002. (DOI.ORG/10.1287/ORSC.13.1.81.542)

ZIMMERMAN, J. Accounting for decision making and control. Boston: Irwin/McGraw Hill, 1997. 